\title{
Association between Growth Hormone-Insulin-Like Growth Factor-1 Axis Gene Polymorphisms and Short Stature in Chinese Children
}

\author{
Yanhong Zhang $\mathbb{D}^{1,2}$ Mei Zhang $\mathbb{D}^{2},{ }^{2}$ Yuntian Chu $\mathbb{D}^{2},{ }^{2}$ Baolan Ji $\mathbb{D}^{2},{ }^{2}$ \\ Qian Shao $\left(\mathbb{D},{ }^{2}\right.$ and Bo Ban $\mathbb{( i D}^{2}$ \\ ${ }^{1}$ Qingdao University, Qingdao 266200, China \\ ${ }^{2}$ Department of Endocrinology, Affiliated Hospital of Jining Medical University, Jining, Shandong 272029, China
}

Correspondence should be addressed to Bo Ban; banbo2011@163.com

Received 21 December 2017; Revised 27 January 2018; Accepted 7 February 2018; Published 4 March 2018

Academic Editor: Paola Di Carlo

Copyright (C) 2018 Yanhong Zhang et al. This is an open access article distributed under the Creative Commons Attribution License, which permits unrestricted use, distribution, and reproduction in any medium, provided the original work is properly cited.

\begin{abstract}
Objective. This study was designed to analyze the association between the growth hormone-insulin-like growth factor-1 (GHIGF-1) axis gene polymorphisms and short stature in Chinese children. Methods. 181 growth hormone deficiency (GHD) patients and 206 normal stature controls were enrolled to attend this study. Five single-nucleotide polymorphisms in the GH receptor (GHR) and 5 SNPs within the GH-signaling pathway were genotyped by matrix-assisted laser desorption/ionization time of flight mass spectrometry. We conducted an association study between these SNPs and the risk of developing short stature. Linkage disequilibrium analysis was performed using Haploview software and the associations of the SNPs frequencies with short stature were analyzed using $X^{2}$ tests. Results. No significant difference was found in gender, weight, height, and BMI between the GHD and control groups, except that the age of GHD group was older than the control one. Allele and genotype frequencies were consistent with those expected from Hardy-Weinberg equilibrium. Compared with the controls, heterozygous genotype frequencies (CT) of rs12515480 and rs6873545 of GHR gene were significantly lower. Genotype frequencies of the other 8 SNPs did not show significant difference between these two groups. Considering a dominant model, an OR $<1$ was observed for genotypes rs12515480 (OR = $0.532, P=0.019)$ and $\mathrm{rs} 6873545(\mathrm{OR}=0.587, P=0.017)$. Conclusions. The heterozygous genotypes of rs12515480 and rs6873545 of GHR gene were associated with decreased risk of GHD in Chinese children.
\end{abstract}

\section{Introduction}

Short stature is a common medical concern in childhood which is characterized by a height more than 2 standard deviations (SD) below the mean for a given age, gender, and population group. Human height depends on complex factors including parents' heredity, genetic mutation, intrauterine infection, nutrition status, hormonal influences, and social environment [1]. Although researchers have identified several causes of short stature which are including familial short stature, hormonal pathologies, systemic diseases, birthday history of intrauterine growth retardation (IUGR), or small for gestational age (SGA) [2]. However, the underlying causes remain unknown in approximately $80 \%$ of patients and are therefore classified as idiopathic short stature (ISS) [3].
Growth hormone deficiency (GHD) is a relatively common disorder with a prevalence of approximately 1 in 4000 during childhood, which is one of the major causes of short stature [4]. Our previous studies showed that the five most common etiological factors of short stature were as follows: GHD (55.56\%), ISS (30\%), hypothyroidism (4.07\%), SGA (2.59\%), and congenital ovarian hypoplasia (2.59\%) [5]. The five most common etiological factors from the study by Sultan et al. [6] were constitutional growth delay (CGD), familial short stature (FSS), malnutrition, coeliac disease, and GHD. Rabbani et al. [7] carried the similar study and also found that GHD was one of the most common causes of short stature. The above information suggests that it would be critical to explore the pathogenesis of GHD. 
Short stature is considered as a genetic origin disease, as shown by identification of genes deficiency associated with short stature, such as short stature homeobox (SHOX), insulin-like growth factor-1 (IGF-1), signal transducer and activator of transcription 5B (STAT5B), insulin-like growth factor binding protein acid subunit (IGFALS), and insulinlike growth factor-1 receptor (IGF1-R). The genome-wide association studies (GWAS) using single-nucleotide polymorphisms (SNPs) analysis have revealed a large number of genetic variants associated with the height, and $75 \%$ of the height could be explained by the inheritance [8], and 180 human genetic loci were found to be related to height, but it can only explain about $10 \%$ of the height variation in the GIANT alliance research [9]. Thus, it suggested that we need to put more efforts into identifying novel genes variants associated with short height.

Traditionally, the growth hormone-insulin-like growth factor-1 (GH-IGF-I) axis is the most important signaling pathway in linear growth, and defects in this axis present as growth hormone deficiencies or IGF-I deficiencies [10]. There were various genes and SNPs associated with short stature, including GHR SNPs (rs10044169, rs12515480, rs4410646, rs6182, and rs6873545), IGF-1R SNPs (rs1976667, rs2684788), IGFALS SNPs (rs17559, rs3751893), and IGFBP3 SNP (rs2132570) [11]. However, the above gene polymorphisms have not been elucidated systematically among Chinese GHD children. Therefore, the aims of this study were to explore the GHD mechanism from the molecular genetic perspective of the GH-IGF-1 axis, to screen SNP locus related to GHD genetic susceptibility, and to analyze the relationships between SNPs of the GH-IGF-1 axis gene and GHD genetic susceptibility.

\section{Subjects and Methods}

2.1. Subjects and Grouping. Short stature patients were voluntary enrolled from the Department of Endocrinology, Affiliated Hospital of Jining Medical University, between May 2013 and April 2016. Normal height control group was recruited from the pediatric clinic during the same period. The subjects were all from the same race. The short stature patients were all evaluated for $\mathrm{GH}$ serum levels after two provocative tests (with arginine or L-dopa or insulin). Inclusion criteria for GHD were as follows: (1) the height was lower than the same age, sex, and the 2 standard deviations (SDs) below the population average height of the same race; (2) peak $\mathrm{GH}$ levels $<10 \mathrm{ng} / \mathrm{ml}$ in both of the two GH stimulation tests; (3) the blood routine examination, liver function, kidney function, thyroid function, and the blood fat were normal; (4) without significant serious psychological and emotional disorders; (5) with normal chromosome and pituitary magnetic resonance; (6) without immunocompetent diseases (i.e., human immunodeficiency virus infections). Familial short stature and physical developmental delay of adolescence were excluded for all subjects. Finally, 181 GHD patients and 206 normal height controls were included in our study. The study was approved by the Human Ethics Committee of the Affiliated Hospital of Jining Medical University (Shandong, China). All of the families of the patients were informed of the aims of the study and the parents gave informed consents.

2.2. Anthropomorphic Measurements. The height was measured by a specially designated individual using the same measuring instrument (produced by Nantong Best Industrial Co. Ltd., Jiangsu, China) in the morning, and the allowable error range is $0.1 \mathrm{~cm}$. Height was expressed as the height standard deviation score (SDS) based on normative values for Chinese children [12]. BMI was calculated as the ratio between body weight in kilograms and height in meters squared. The stage of puberty was assessed by physical examinations according to Tanner and Whitehouse [13]. The following criteria were considered as prepuberty [14, 15]: girls with no breast development and no pubic hair and boys with a testicular volume of less than $4 \mathrm{ml}$ and no pubic hair. The bone age (BA) was measured by taking X-ray of the left hand, including the hand bone, wrist, and radial ulnar stem $3-4 \mathrm{~cm}$. The same specially assigned investigator scanned the image and evaluated the BA according to the Greulich-Pyle method [16].

2.3. Laboratory Measurements. To assess GH secretion, Ldopa (Levodopa Tablets ${ }^{\circledR}$, He Feng, Guang Xi, China, body weight more than $30 \mathrm{~kg}, 500 \mathrm{mg}$ of levodopa; less than $30 \mathrm{~kg}$, $250 \mathrm{mg}$ of levodopa) and insulin (Insulin Injection ${ }^{\circledR}$, Wan Bang, Jiang Su, China, $0.1 \mathrm{U} / \mathrm{kg}$ ) were administered orally or subcutaneously injected after overnight fasting. Blood samples were collected $0,30,60,90$, and $120 \mathrm{~min}$ later to obtain the serum GH concentration for each time point. $\mathrm{GH}$ was measured using an chemiluminescence method (ACCESS2, Beckman Coulter, USA) with an analytical sensitivity of $0.010 \mathrm{ug} / \mathrm{L}$. Serum IGF-1 and IGFBP-3 levels were measured by chemiluminescence immunometric method (DPC IMMULITE 1000 analyzer, SIEMENS, Germany) with intra- and interassay CV for IGF-1 of $3.0 \%$ and $6.2 \%$, respectively, and intra- and interassay CV for IGFBP-3 of $4.4 \%$ and $6.6 \%$, respectively. The liver functions, including alanine aminotransferase (ALT), AST and gamma-glutamyl transferase (GGT), kidney function, including $\mathrm{Cr}$, blood urea nitrogen (BUN), and UA, lipid profiles, TC, high density lipoprotein-cholesterol (HDL-C), LDL-C, triglycerides (TG), and fasting plasma glucose (FPG) were tested by a biochemical autoanalyzer (cobas c 702, Roche; Shanghai, China); thyroid function, including free T3 (FT3), free T4 (FT4) and thyroid stimulating hormone (TSH), gonadotropin, cortisol rhythm, and adrenocorticotropic hormone (ACTH) were tested by a luminescence immunoassay system (cobas e 602, Roche; Shanghai, China).

2.4. SNP Selection and Genotyping. Genomic DNA was isolated from whole blood using the DNeasy Blood and Tissue Kit (Qiagen). The following genes and SNPs were selected for analysis: GHR SNPs (rs10044169, rs12515480, rs4410646, rs6182, and rs6873545), IGF-1R SNPs (rs1976667 and rs2684788), IGFBP-3 SNP (rs2132570), and IGFALS SNPs (rs17559 and rs3751893). After the polymerase chain reaction (PCR) and purifying of the product, we used matrix-assisted 
TABLE 1: The clinical characteristics of all study participants.

\begin{tabular}{lcc}
\hline Characteristic & Control & GHD \\
\hline$N$ & 206 & 181 \\
Gender $(n, \%)$ & & \\
$\quad$ Female & $96(46.6 \%)$ & $51(28.2 \%)$ \\
$\quad$ Male & $110(53.4 \%)$ & $130(71.8 \%)$ \\
Age $(\mathrm{y})$ & $7.5 \pm 3.2$ & $10.2 \pm 3.1^{*}$ \\
Height $(\mathrm{cm})$ & $129.9 \pm 20.6$ & $127.9 \pm 16.1$ \\
Weight $(\mathrm{Kg})$ & $31.3 \pm 15.2$ & $29.5 \pm 11.0$ \\
BMI $\left(\mathrm{Kg} / \mathrm{m}^{2}\right)$ & $17.5 \pm 3.7$ & $17.5 \pm 3.1$ \\
\hline
\end{tabular}

Data are mean \pm standard deviation. $\mathrm{BMI}=$ body mass index. ${ }^{*} P<0.05$ is considered significant.

laser desorption ionization time of flight mass spectrometry (MALDI-TOF MS) methods to detect the sample.

2.5. Statistical Analysis. All of the statistical analyses were performed with the $\mathrm{R}$ statistical software (https://www.rproject.org) and EmpowerStats (https://www.r-project.org, $\mathrm{X} \& \mathrm{Y}$ solutions, Inc. Boston MA). Normally distributed variables were expressed as mean \pm standard deviation (SD); qualitative data were expressed as numbers and percentage $(n$, $\%)$. An independent samples $t$-test was used to compare continuous variables between the two groups. The chi-squared test was used to analyze differences between the control and GHD groups in SNP genotypes of the GH-signaling related genes, alleles, and dominant and recessive modes. Linkage disequilibrium calculation and haplotype frequencies determination were performed with the Haploview software (Center for Human Genetic Research, Massachusetts General Hospital, and the Broad Institute of Harvard and MIT). $P$ values (2-tailed) less than 0.05 were considered statistically significant.

\section{Results}

3.1. Comparison of Clinical Characteristics of Short Stature Patients and Normal Control Group. In this study, we enrolled total 387 subjects, including $181 \mathrm{GHD}$ patients (GHD group) and 206 subjects with normal height (control group). The clinical characteristics of the subjects are shown in Table 1 . The mean age in control group was younger than that in the GHD group $(P<0.001)$. The height of controls was slightly higher than that in GHD group $(P=0.287)$. In addition, the BMI in GHD group was slightly lower than that in control group $(P=0.980)$.

3.2. The Genotype Distribution Frequency of Ten SNPs Loci of GH-IGF-1 Related Genes in the Study Population. Table 2 summarizes the genotype distribution frequency of ten SNPs loci of GH-IGF-1 related genes in the study population. The Hardy-Weinberg equilibrium test showed no significant difference in ten SNP loci between the GHD group and the normal population $(P>0.05)$. Furthermore, we compared the differences in allele frequencies distribution of ten reported SNPs including GHR SNPs (rs10044169, rs12515480, rs4410646, rs6182, and rs6873545), IGF-1R SNPs (rs1976667 and rs2684788), IGFBP-3 SNP rs2132570, and IGFALS SNPs (rs17559 and rs3751893) in GHD and control group. The results showed that compared with the control group, heterozygous genotypes of rs12515480 and rs6873545 were significantly lower in GHD group $(P=0.040$ and $P=0.046$, resp.). However, no significant differences in allele frequencies distribution of the other 8 SNPs (rs10044169, rs4410646, rs6182, rs1976667, rs2684788, rs2132570, rs17559, and rs3751893) were observed in GHD and control group.

3.3. Association of GHR Polymorphism with Susceptibility to GHD. In order to analyze the association of GH-IGF axis related gene polymorphisms with the risk of short stature, we assessed the association between the rs12515480 and rs6873545 SNPs and the risk of short stature using three genetic models (codominants, dominant and recessive) by unconditional logistic-regression analysis. As shown in Table 3, the crude analysis revealed that the genotype "CT+TT" in rs12515480 from GHR was associated with a decreased risk of GHD under the dominant model $(\mathrm{OR}=$ 0.587, 95\% CI: $0.364-0.947, P=0.029)$. Also, we found that the significance still existed after adjusting for gender and age (OR $=0.532,95 \%$ CI: $0.314-0.900, P=0.019)$. Similarly, the genotype "CC+CT" in rs6873545 from GHR was associated with a decreased risk of GHD under the dominant model $(\mathrm{OR}=0.657,95 \% \mathrm{CI}: 0.445-0.971, P=0.035)$. And the significance still existed after adjusting for gender and age (OR $=0.587,95 \%$ CI: 0.380-0.908, $P=0.017)$.

\section{Discussion}

In this study, we assessed the association between ten SNPs within GH-signaling and the risk of short stature in China. We found that the "CT+TT" in rs12515480 and "CC+CT" in rs6873545 from GHR was associated with a decreased risk of GHD under the dominant model, presented to be a protective effect on the incidence of GHD.

The GH-IGF-I axis possesses various effects involving height growth, cardiac function, and behavioral psychology [17]. Many factors could affect the role of this axis [1], and the heredity is especially notable [8]. The growth hormone receptor (GHR) exon3 deleted/full-length (d3/fl) polymorphism has been suggested to affect GH sensitivity. Previous studies have confirmed that the GHR $\mathrm{d} 3 / \mathrm{fl}$ polymorphism can be studied by TaqMan SNP rs6873545 genotyping [18]. The homozygous mutation of the $\mathrm{d} 3-\mathrm{GHR}$ showed a poor response to $\mathrm{GH}$ treatment than carriers of the fl-GHR in GHD adult [19]. However, another research did not show any association between the d3-GHR genotype and decreased sensitivity to $\mathrm{GH}$ in ISS children [20]. However, in our study, homozygous mutation of rs6873545 was rare and the heterozygous genotype was relatively higher within GHD group. Furthermore, the correlation analysis showed that "CC+CT" in rs6873545 was negatively related to the GHD. The underlying mechanism is not yet known and needs to be further explored by expanding the sample size or gene function study. 
TABLE 2: Genotype distributions of GH-IGF axis related gene included in this study.

\begin{tabular}{|c|c|c|c|c|}
\hline Gene SNPs & Genotype & $\begin{array}{c}\text { Control } \\
(n=206)\end{array}$ & $\begin{array}{c}\text { GHD } \\
(n=181)\end{array}$ & HWE $P$ value \\
\hline \multicolumn{5}{|l|}{ GHR } \\
\hline \multirow{3}{*}{ rs10044169 } & $\mathrm{AA}$ & $161(78.2 \%)$ & $148(81.8 \%)$ & 0.751 \\
\hline & $\mathrm{AC}$ & $43(20.9 \%)$ & $31(17.1 \%)$ & \\
\hline & CC & $2(1.0 \%)$ & $2(1.1 \%)$ & \\
\hline \multirow{3}{*}{ rs12515480 } & $\mathrm{CC}$ & $161(78.2 \%)$ & $158(87.3 \%)$ & 0.651 \\
\hline & CT & $43(20.9 \%)$ & $22(12.2 \%)^{*}$ & \\
\hline & $\mathrm{TT}$ & $2(1.0 \%)$ & $1(0.4 \%)$ & \\
\hline \multirow{3}{*}{ rs4410646 } & $\mathrm{AA}$ & $113(36.0 \%)$ & $81(35.4 \%)$ & 0.330 \\
\hline & $\mathrm{AC}$ & $152(48.4 \%)$ & $120(52.4 \%)$ & \\
\hline & CC & $49(15.6 \%)$ & $28(12.2 \%)$ & \\
\hline \multirow{3}{*}{ rs6182 } & GG & $252(80.3 \%)$ & $189(82.5 \%)$ & 0.884 \\
\hline & GT & $57(18.2 \%)$ & $38(16.6 \%)$ & \\
\hline & $\mathrm{TT}$ & $4(1.3 \%)$ & $2(0.9 \%)$ & \\
\hline \multirow{3}{*}{ rs6873545 } & TT & $217(69.1 \%)$ & 177 (77.3\%) & 0.635 \\
\hline & CT & $91(29.0 \%)$ & $46(20.1 \%)^{*}$ & \\
\hline & CC & $6(1.9 \%)$ & $6(2.6 \%)$ & \\
\hline \multicolumn{5}{|l|}{ IGF1R } \\
\hline \multirow{3}{*}{ rs1976667 } & $\mathrm{AA}$ & $115(55.8 \%)$ & $83(45.9 \%)$ & 0.957 \\
\hline & $\mathrm{AG}$ & 77 (37.4\%) & $83(45.9 \%)$ & \\
\hline & GG & $14(6.8 \%)$ & $15(8.3 \%)$ & \\
\hline \multirow{3}{*}{ rs2684788 } & CC & $55(26.7 \%)$ & $49(27.1 \%)$ & 0.886 \\
\hline & CT & $96(46.6 \%)$ & $88(48.6 \%)$ & \\
\hline & TT & $55(26.7 \%)$ & $44(24.3 \%)$ & \\
\hline \multicolumn{5}{|l|}{ IGFBP3 } \\
\hline \multirow{3}{*}{ rs 2132570} & GG & $134(65.0 \%)$ & $121(66.9 \%)$ & 0.351 \\
\hline & GT & $67(32.5 \%)$ & $55(30.4 \%)$ & \\
\hline & $\mathrm{TT}$ & $5(2.4 \%)$ & $5(2.8 \%)$ & \\
\hline \multicolumn{5}{|l|}{ IGFALS } \\
\hline \multirow{3}{*}{ rs17559 } & GG & $132(64.1 \%)$ & $113(62.4 \%)$ & 0.931 \\
\hline & GA & $68(33.0 \%)$ & $61(33.7 \%)$ & \\
\hline & $\mathrm{AA}$ & $6(2.9 \%)$ & $7(3.9 \%)$ & \\
\hline \multirow{3}{*}{ rs3751893 } & GG & $116(56.3 \%)$ & $103(56.9 \%)$ & 0.195 \\
\hline & GA & $77(37.4 \%)$ & $58(32.0 \%)$ & \\
\hline & $\mathrm{AA}$ & $13(6.3 \%)$ & $20(11.0 \%)$ & \\
\hline
\end{tabular}

GHD, growth hormone deficiency; SNP, single-nucleotide polymorphism; ${ }^{*} P<0.05$ is considered significant.

TABLE 3: Genotype effects of rs12515480 and rs6873545 variation.

\begin{tabular}{lccccc}
\hline SNPs & Alleles & OR & $95 \%$ CI & $\begin{array}{c}\text { Best-fitting } \\
\text { model }\end{array}$ & $P$ value \\
\hline rs12515480 & $C / T$ & 0.532 & $(0.314,0.900)$ & Dominant & 0.019 \\
rs6873545 & $T / C$ & 0.587 & $(0.380,0.908)$ & Dominant & 0.017 \\
\hline
\end{tabular}

GHD, growth hormone deficiency; SNP, single-nucleotide polymorphism; OR, odds ratio; CI, confidence interval. The model was adjusted for age and sex.

There were few studies about the relationship between the rs12515480 polymorphism and short stature. Zeng et al. [21] found that GHR gene rs12515480 (C/T) polymorphisms were not associated with idiopathic short stature in Yao nationality children of Guangxi province in China. However, in our study, heterozygous genotype of rs12515480 was negatively related to the GHD. The rs12515480 locus is located in the intron region of GHR gene, and we did not find related studies investigating the structure and function of the encoded protein at this locus. Further studies should be taken to explore the relationship and the mechanism.

In our study, the rest $8 \mathrm{SNPs}$ within $\mathrm{GH}$-signal axis were not related to genetic susceptibility of GHD. In consistent with the previous study [22], the result showed that there 
were no differences in height of SDS, IGF-1, IGFBP-3, and ALS between carriers and nonallele of the common IGFALS gene loci rs17559 and rs3751893 among normal control and ISS children. They suggested that heterozygous IGFALS gene variants could be responsible for short stature in a subset of ISS children with diminished levels of IGF-1, IGFBP3 , and ALS. However, in our study, we did not find any difference in the above gene polymorphisms and genetic susceptibilities to GHD, whereas our results were inconsistent with other studies. Yu et al. [23] found that three SNPs of GHR (rs6182, rs4410646, and rs10044169) are related to genetic susceptibility to ISS. For rs6182 (G/T), genotypes TT and GT showed a decreased risk under $\mathrm{T}$ dominant model (OR = 0.624). Genotype AA of rs4410646 presented a lower risk under $\mathrm{C}$ dominant model $(\mathrm{OR}=0.674)$. "CC+CA" genotypes of rs10044169 were associated with a decreased risk of ISS under $\mathrm{C}$ dominant model ( OR = 0.649). Yang et al. [24] revealed that the rs1976667 and rs2684788 loci were significantly associated with genetic susceptibility to ISS. The $G$ allele at the rs2684788 locus was significantly associated with genetic susceptibility to ISS, showing G dominant inheritance. The rs1976667 and rs2684788 loci of the human IGF-1R gene are likely associated with different genetic susceptibilities to ISS in males and females. But the above studies were focused on ISS children; the underlying principle is unclear and needs to be further explored in the subjects with GHD.

There are several limitations to this study. On the one hand, in this correlation study, we could not conclude the causal relationship. On the other hand, other factors that may affect the study have not been adjusted, such as parent height. Furthermore, it is regrettable that some influencing factors including congenital infection (i.e., toxoplasma gondii infection) and nutrition status during fetal and childhood are missing or incomplete. Therefore, subsequent large samples and prospective and comprehensive studies might be needed to verify the results of this study.

In conclusion, this study showed that heterozygous genotypes of rs12515480 and rs6873545 of GHR gene were associated with decreased risk of developing GHD in Chinese children. However, further studies should be conducted to explore and verify the findings and mechanism of the study.

\section{Conflicts of Interest}

The authors declare that there are no conflicts of interest.

\section{Authors' Contributions}

Yanhong Zhang conducted data collection and data analysis. Mei Zhang, Bo Ban, and Qian Shao have contributed to the design of the study. Yuntian Chu focused on the part of data analysis, while Baolan Ji worked on the document revision. All the authors reviewed the paper.

\section{Acknowledgments}

This research was carried out at Affiliated Hospital of Jining Medical College and was supported by grants from the
Natural Science Foundation of Shandong Province (no. ZR2014HP058) and Health and Family Planning Commission of Shandong Province (no. 2014-032).

\section{References}

[1] C. P. Hawkes and A. Grimberg, "Insulin-like growth factor-I is a marker for the nutritional state," Pediatric Endocrinology Reviews, vol. 13, no. 2, pp. 499-511, 2015.

[2] L. A. Waldman and D. J. Chia, "Towards identification of molecular mechanisms of short stature," International Journal of Pediatric Endocrinology, vol. 2013, no. 1, 19 pages, 2013.

[3] P. Cohen, A. D. Rogol, C. L. Deal et al., "Consensus statement on the diagnosis and treatment of children with idiopathic short stature: a summary of the Growth Hormone Research Society, the Lawson Wilkins Pediatric Endocrine Society, and the European Society for Paediatric Endocrinology Workshop," The Journal of Clinical Endocrinology \& Metabolism, vol. 93, no. 11, pp. 4210-4217, 2008.

[4] NICE, "Human growth hormone (somatropin) for the treatment of growth failure in children," 2010, http://www.nice.org .uk/guidance/ta188.

[5] B. Ban, H. Pan, Q. Shao et al., "Etiologic and diagnostic analysis of 270 children with short stature in China Jining region," Chinese Journal of Diagnostics (Electronic Edition), vol. 2, no. 2, pp. 100-104, 2014.

[6] M. Sultan, M. Afzal, S. M. Qureshi et al., "Etiology of short stature in children," Journal of the College of Physicians and Surgeons Pakistan, vol. 18, no. 8, pp. 493-497, 2008.

[7] M. W. Rabbani, W. I. Khan, A. B. Afzal, and W. Rabbani, "Causes of short stature identified in children presenting at a tertiary care hospital in Multan Pakistan," Pakistan Journal of Medical Sciences, vol. 29, no. 1, pp. 53-57, 2013.

[8] J. A. Yang, B. Benyamin, B. P. McEvoy et al., "Common SNPs explain a large proportion of the heritability for human height," Nature Genetics, vol. 42, no. 7, pp. 565-569, 2010.

[9] H. L. Allen, K. Estrada, G. Lettre et al., "Hundreds of variants clustered in genomic loci and biological pathways affect human height," Nature, vol. 467, pp. 832-838, 2010.

[10] M. H. Aguiar-Oliveira, A. H. O. Souza, C. R. P. Oliveira, V. C. Campos, L. A. Oliveira-Neto, and R. Salvatori, "MECHANISMS IN ENDOCRINOLOGY: The multiple facets of GHRH/GH/IGF-I axis: lessons from lifetime, untreated, isolated GH deficiency due to a GHRH receptor gene mutation," European Journal of Endocrinology, vol. 177, no. 2, pp. R85-R97, 2017.

[11] J. M. Wit, W. Oostdijk, M. Losekoot, H. A. Van Duyvenvoorde, C. A. L. Ruivenkamp, and S. G. Kant, "MECHANISMS IN ENDOCRINOLOGY: Novel genetic causes of short stature," European Journal of Endocrinology, vol. 174, no. 4, pp. R145R173, 2016.

[12] H. Li, C. Y. Ji, X. N. Zong, and Y. Q. Zhang, "Height and weight standardized growth charts for Chinese children and adolescents aged 0 to 18 years," Zhonghua Er Ke Za Zhi, vol. 47, no. 7, pp. 487-492, 2009.

[13] J. M. Tanner and R. H. Whitehouse, "Clinical longitudinal standards for height, weight, height velocity, weight velocity, and stages of puberty," Archives of Disease in Childhood, vol. 51, no. 3, pp. 170-179, 1976.

[14] W. A. Marshall and J. M. Tanner, "Variations in pattern of pubertal changes in girls," Archives of Disease in Childhood, vol. 44, no. 235, pp. 291-303, 1969. 
[15] W. A. Marshall and J. M. Tanner, "Variations in the pattern of pubertal changes in boys," Archives of Disease in Childhood, vol. 45, no. 239, pp. 13-23, 1970.

[16] W. W. Greulich and S. I. Pyle, "Radiographic atlas of skeletal development of the hand and wrist," The American Journal of the Medical Sciences, vol. 238, no. 3, p. 393, 1959.

[17] D. E. Sandberg and M. Gardner, "Short stature: is it a psychosocial problem and does changing height matter?" Pediatric Clinics of North America, vol. 62, no. 4, pp. 963-982, 2015.

[18] J. Wan, G. Atzmon, D. Hwang, N. Barzlai, J. Kratzsch, and P. Cohen, "Growth hormone receptor (GHR) exon 3 polymorphism status detection by dual-enzyme-linked immunosorbent assay (ELISA)," The Journal of Clinical Endocrinology \& Metabolism, vol. 98, no. 1, pp. E77-E81, 2013.

[19] C. A. M. Glad, E. J. L. Barbosa, H. F. Nyström et al., "SNPs within the GH-signaling pathway are associated with the early IGF1 response to GH replacement therapy in GHD adults," European Journal of Endocrinology, vol. 170, no. 1, pp. 101-107, 2014.

[20] G. Hellgren, C. A. Glad, B. Jonsson, G. Johannsson, and K. Albertsson-Wikland, "The growth hormone receptor exon 3deleted/full-length polymorphism and response to growth hormone therapy in prepubertal idiopathic short children," Growth Hormone \& IGF Research, vol. 25, no. 3, pp. 127-135, 2015.

[21] T. Zeng, T. Z. Yan, N. Tang et al., "Relationship between growth hormone receptor gene polymorphism and idiopathic short stature in Guangxi Yao nationality children," Chinese Journal of Child Health Care, vol. 23, no. 7, pp. 699-709, 2015.

[22] H. M. Domene, P. A. Scaglia, A. S. Martinez et al., "Heterozygous IGFALS gene variants in idiopathic short stature and normal children: impact on height and the IGF system," Hormone Research in Paediatrics, vol. 80, no. 6, pp. 413-423, 2014.

[23] Y. Yu, W. Wang, Y. Wang et al., "Associations of single nucleotide polymorphisms of growth hormone receptor with susceptibility to idiopathic short stature," Journal of Shanghai Jiaotong University (Medical Science), vol. 31, no. 7, pp. 932-936, 2011.

[24] Y. Yang, H. Huang, W. Wang, L. Yang, L. L. Xie, and W. Huang, "Association of insulin growth factor-1 receptor gene polymorphisms with genetic susceptibility to idiopathic short stature," Genetics and Molecular Research, vol. 12, no. 4, pp. 4768-4779, 2013. 


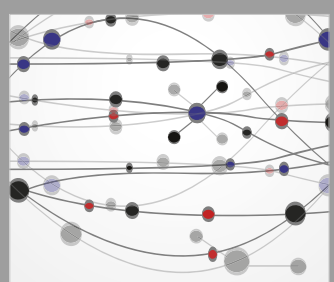

The Scientific World Journal
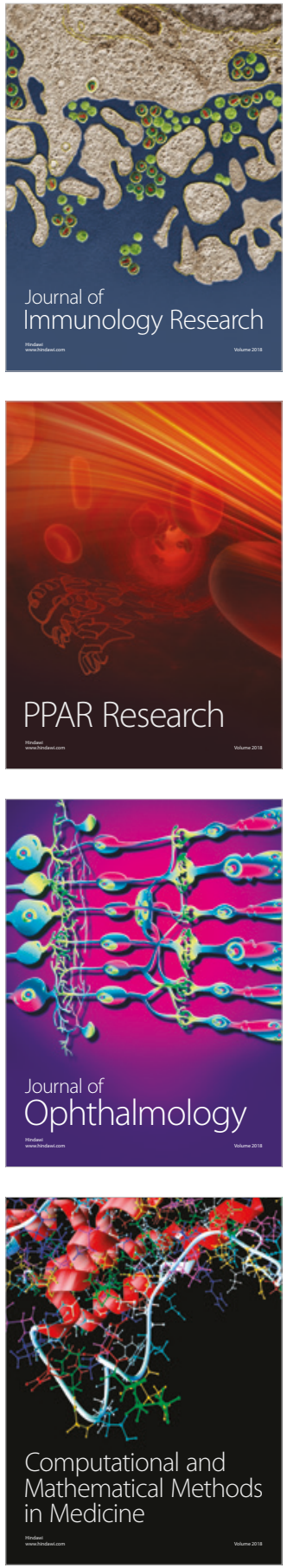

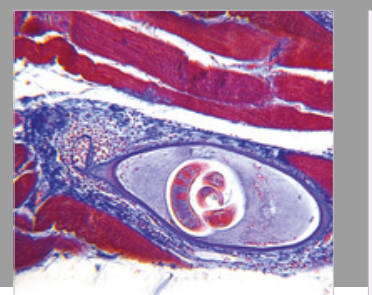

Gastroenterology Research and Practice

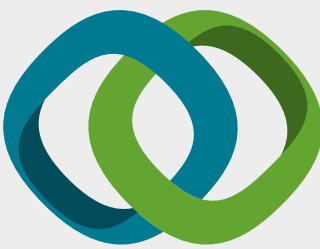

\section{Hindawi}

Submit your manuscripts at

www.hindawi.com
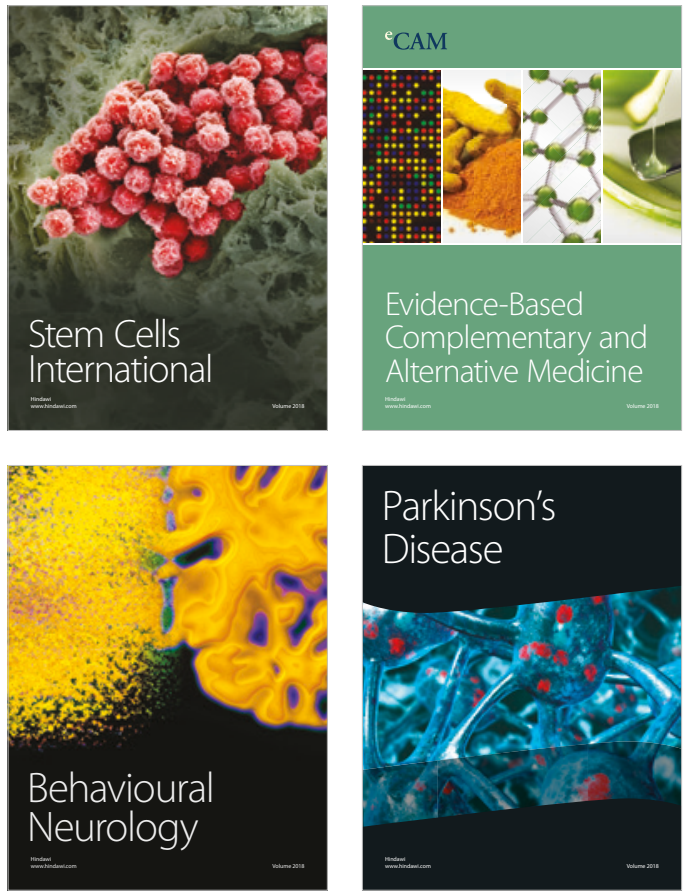

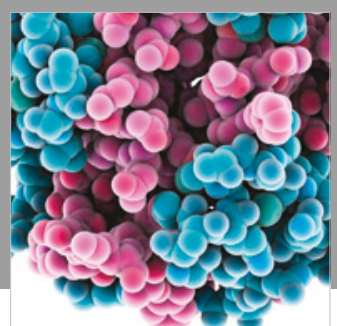

ournal of

Diabetes Research

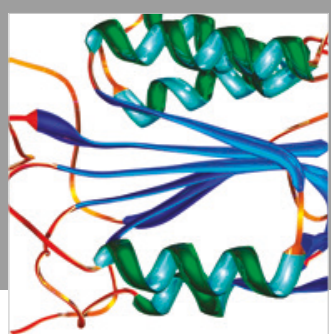

Disease Markers
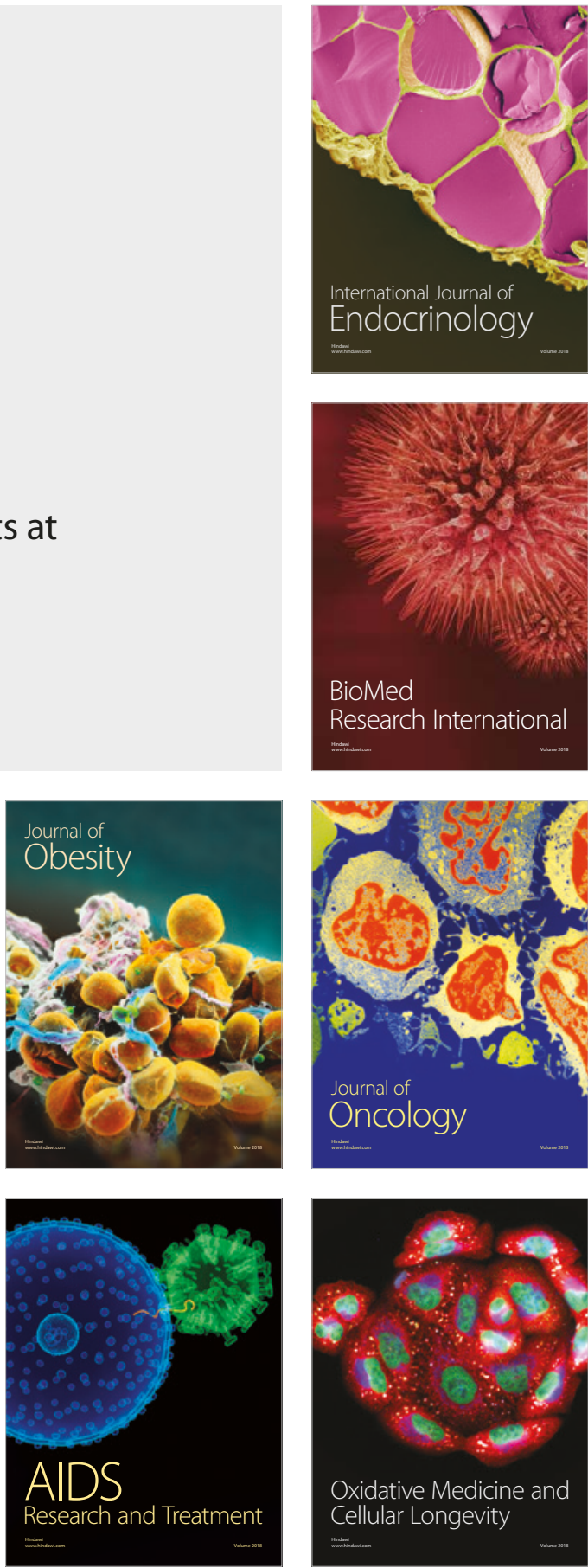